\title{
Subfoveal choroidal thickness at age 9 years in relation to clinical and perinatal characteristics in the population-based Generation R Study
}

\author{
Kevser Zehra Biyik, ${ }^{1,2,3}$ (D) Jan Willem L. Tideman, ${ }^{1}$ (D) Jan Roelof Polling, ${ }^{1,4}$ Gabriëlle H. S. Buitendijk, \\ Vincent V. W. Jaddoe, ${ }^{5}$ Michael Larsen ${ }^{2}$ and Caroline C. W. Klaver ${ }^{1,6}$ \\ ${ }^{1}$ Department of Ophthalmology and Department of Epidemiology, Erasmus Medical Center, Rotterdam, The Netherlands \\ ${ }^{2}$ Department of Ophthalmology, Rigshospitalet, Glostrup, Denmark \\ ${ }^{3}$ Faculty of Health Sciences, University of Copenhagen, Copenhagen, Denmark \\ ${ }^{4}$ Department of Orthoptics, School of Applied Science Utrecht, Utrecht, The Netherlands \\ ${ }^{5}$ Department of Pediatrics, Erasmus Medical Center, Rotterdam, Netherlands \\ ${ }^{6}$ Department of Ophthalmology, Radboud UMC, Nijmegen, The Netherlands
}

\begin{abstract}
.
Purpose: To assess the association between clinical and perinatal characteristics and subfoveal choroidal thickness in 9-year-old children.

Methods: The study included data from the population-based Generation $\mathbf{R}$ cohort, whose participants underwent cycloplegic refractometry, ocular biometry, height, weight and subfoveal choroidal thickness measurements using a sweptsource optical coherence tomography (SS-OCT) instrument. Birth parameters were obtained using medical records. Statistical analyses were performed using multivariate regression models adjusted for age, ethnicity and sex.

Results: A total of 1018 children (52.5\% girls, $47.5 \%$ boys) with a mean age of $9.9 \pm 0.3$ years and a mean cycloplegic spherical equivalent refraction of $0.80 \pm 1.1$ $D$ in boys and $0.81 \pm 1.4$ in girls were eligible for analysis. The subfoveal choroid was $17 \mu \mathrm{m}$ thicker in girls $(298 \pm 60.6 \mu \mathrm{m})$ than in boys $(281 \pm 55.0 \mu \mathrm{m} ; \mathrm{p}<0.001)$, a difference of $9.1 \mu \mathrm{m}$ persisting after adjustment for age, ethnicity and axial length $(p=0.017)$. Subfoveal choroidal thickness decreased with increasing ocular axial length $(-16.2 \mu \mathrm{m} / \mathrm{mm}, 95 \% \mathrm{CI}-21.2$ to $-12.4, \mathrm{p}<0.001)$ and with increasing myopic refraction $(-10.0 \mu \mathrm{m} / \mathrm{D}, 95 \%$ CI $6.8-13.1 ; \mathrm{p}<0.001$, adjusted for age, ethnicity, axial length and sex) while it increased with increasing body height $(1.3 \mu \mathrm{m} /$ $\mathrm{cm}, 95 \% \mathrm{CI} 0.8$ to $1.9, \mathrm{p}<0.001$ ). Additionally, choroidal thickness increased with increasing birthweight $(13.0 \mu \mathrm{m} / \mathrm{kg} ; 95 \%$ CI 0.006-0.020; $p<0.001)$ and increasing size for gestational age $(8.2 \mu \mathrm{m} / \mathrm{kg} ; 95 \%$ CI $4.6-11.8 ;$ p $<0.001)$. Smoking up until the time that pregnancy became known was associated with a thinner choroid $(p=0.016)$. There was no detectable effect of alcohol consumption. The distributions of axial length, refraction and choroidal thickness were narrower than in older populations.

Conclusion: The subfoveal choroid was thicker in girls than in boys, and higher body height, higher birthweight and larger size for gestational age were associated with a thicker subfoveal choroid. The implications of these findings for myopia development need further evaluation in longitudinal studies.
\end{abstract}

Key words: children - choroidal thickness - optical coherence tomography - population study

Acta Ophthalmol.

( 2019 Acta Ophthalmologica Scandinavica Foundation. Published by John Wiley \& Sons Ltd

doi: $10.1111 /$ aos. 14178

\section{Introduction}

Myopia or short-sightedness affects more than $50 \%$ of the population in several eastern Asian countries such as Japan and Singapore (Fredrick 2002; Resnikoff et al. 2008). Myopia is a failure of emmetropization, the process that balances the refractive components of the eye with the axial length of the eye to place the focus of distant objects on the retina without accommodation (Nickla \& Wallman 2010). Axial length is closely related to both the spherical equivalent refraction of the eye, the thickness of the choroid and the thickness of the sclera. The choroid is suspected of playing a mediating role in the signalling chain that begins with the registration of the quality of the image cast upon the retina and ends with a change of the position of the retina effected by a change in thickness and rigidity of the sclera and the choroid (Goldenberg et al. 2012; Zhu et al. 2005; Read et al. 2013a,b). With the introduction of optical coherence tomography, anatomical characteristics of the choroid have become amenable to objective assessment. The present report describes associations of choroidal thickness with current and past ocular and systemic characteristics in a cross-sectional study. 


\section{Methods}

\section{Study design and participants}

This study was embedded in the Generation R Study, a population-based prospective cohort study of pregnant women and their children in Rotterdam, the Netherlands. The complete methodology has been described elsewhere (Kooijman et al. 2016). Briefly, a total of 9778 pregnant women were included in the study, and children were born between April 2002 and January 2006. The children were invited at age 9 years for examination by trained nurses at the research centre. The study protocol was approved by the Medical Ethical Committee of the Erasmus Medical Centre, Rotterdam (MEC 217.595/2002/20), and written informed consent was obtained from all participants. The research was conducted according to the Declaration of Helsinki.

\section{Procedures}

Study recruitment was based on inviting pregnant women who attended public health care clinics within a given geographic region during a specific period. A total of 1422 children of the population-based Generation $\mathrm{R}$ birth cohort from Rotterdam were eligible for examination between 1 June 2013 and 1 October 2014 using a sweptsource optical coherence tomography instrument (SS-OCT; DRI-OCT-1, Topcon Corp., Tokyo, Japan) for imaging of the posterior segment of the eye. The methods of choroidal thickness measurement and data analysis were very similar to those used in the Copenhagen Child Cohort 2000 (CCC2000) Eye Study (Li et al. 2014a,b), with the exception that Generation $\mathrm{R}$ used cycloplegia and swept-source optical coherence tomography for imaging, whereas the CCC2000 used noncycloplegic refractioning and spectral-domain optical coherence tomography. Scans were acquired by study nurses and other trained staff, who were instructed to reject scans of poor quality and to continue until optimum quality had been achieved in a given patient. All scans were manually segmented and subfoveal choroidal thickness measured by the same person (KZBK) who also rejected scans where the boundaries of the subfoveal choroid could not be reliably identified. Subfoveal choroidal thickness was measured on seven-line horizontal transfoveal B scans, using the instrument manufacturer's proprietary software (3DOCT-2000, version 4.30.003.02, Tokyo, Japan). Additional scans available for the grader's visual assessment of the chorioscleral boundary included a fovea-centred array of 128 B scans each made up of 512 A scans covering an area of $12.0 \mathrm{~mm} \times 9.0 \mathrm{~mm}$. The foveal centre was assumed to be found on the scan line with the deepest foveal depression and the most prominent bottom reflex (Fig. 1). Choroidal thickness was measured as the distance between Bruch's membrane and the inner surface of the chorioscleral border, both determined by a trained operator (KZBK), who manually adjusted a software caliper feature that was precalibrated by the manufacturer. Intragrader repeatability was measured as the intraclass correlation coefficient (ICC) and found to be 0.983 .

Objective refraction was measured using a hand-held automated refractometer (Retinomax-3, Right Mfg. Co., Ltd., Tokyo, Japan). Pupils were dilated using cyclopentolate $1 \%$ eye drops, two drops per eye as a standard and three in eyes with brown irides. Spherical equivalent refraction measurements and OCT measurements were made at least $30 \mathrm{~min}$ later. The pupil diameter was $\geq 6 \mathrm{~mm}$ at the time of the measurement. SE was calculated as the average spherical correction + half of the cylinder value. Only scans from right eyes were analysed, to avoid having data sets where some

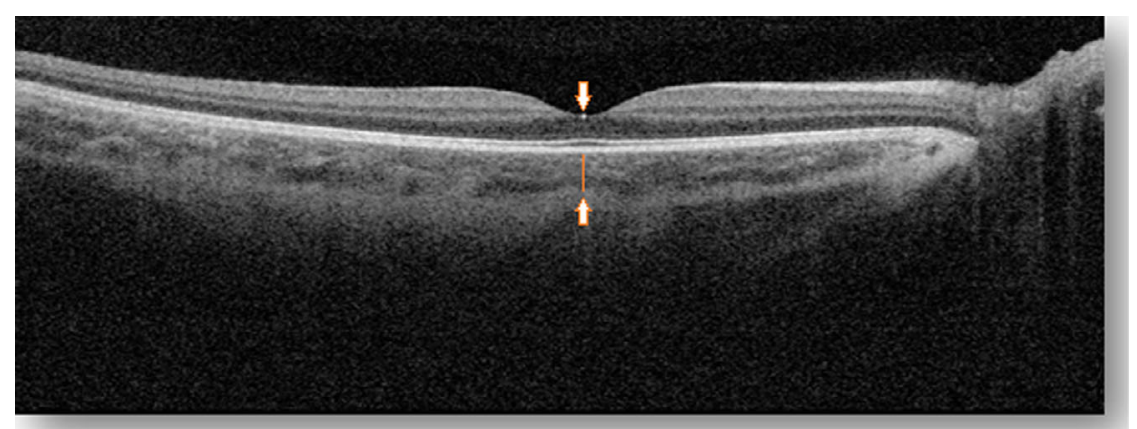

Fig. 1. Horizontal transfoveal optical coherence tomography scan from the right eye of a healthy study participant. The centre of the fovea was identified as the lowest point of the foveal depression (arrow pointing down). Subfoveal choroidal thickness (red line) was measured as the distance between Bruch's membrane and the inner surface of the choroidoscleral border (above arrow pointing up). When irregular scans were found, such as a sloping retina, the distance was measured at a right angle to Bruch's membrane. (age, weight, height etc.), whereas others are eye-specific (refraction, axial length), yet linked by identical genetics, etc. Weight was measured in lightweight clothes and without shoes using a mechanical scale. Height was measured in the standing position using a Harpenden stadiometer (Holtain Limited, Wales, UK). Body mass index (BMI) was calculated using the formula BMI $=$ weight $(\mathrm{kg}) /\left(\right.$ length $(\mathrm{m})^{2}$. Birth parameters were obtained from medical records (Kooijman et al. 2016). Gestational age-adjusted standard deviation birthweight scores were North European birth cohort (Niklasson et al. 1991).

Optical coherence tomography was made in 1422 children. Exclusion criteria included missing OCT scans and scans of poor quality $(n=333)$, missing axial length measurement $(n=3)$, missing ethnicity information $(n=29)$, missing gestational age $(n=5)$, missing birthweight $(n=2)$ and being a twin $(n=32)$. This led to 404 children being excluded from the analysis. Only right eyes were included. Cycloplegic spherical equivalent refraction was available in 778 children.

\section{Statistical analysis}

Demographic data were presented as means and standard deviations for continuous data. Comparisons between sexes were made using Student's twotailed $t$-test. One-way ANOVA was used to compare demographic characteristics between subgroups. The associations between subfoveal choroidal parameters are the same for both eyes based on reference charts from a large 
thickness and relevant parameters were assessed using a general linear model. Homogeneity of variance of subfoveal choroidal thickness was assessed using Levene's test. Intragrader repeatability was evaluated by calculating the intraclass correlation coefficient using mixed models between repeated measurements of the horizontal choroidal scan in 130 randomly chosen children. Multivariate regression analysis was performed including parameters that were significantly associated with subfoveal choroidal thickness in univariate analyses and, invariably, parameters that are considered obligatory (age, ethnicity, sex). Because birthweight, gestational age and size for gestational age are highly correlated, adjustment for each of these parameters was not applied in one and the same model. The level of statistical significance was set to $\mathrm{p} \leq 0.05$, and effect estimates are presented with $95 \%$ confidence intervals $(95 \%$ CI). Statistical analyses were performed using the IBM-SPSS statistics software package version 21.0 (IBM-SPSS Inc., Chicago, IL, USA).

\section{Results}

This cross-sectional study included 1018 children $(52.5 \%$ girls, $47.5 \%$ boys; Fig. 2) with a mean age of $9.9 \pm 0.3$ years and a mean cycloplegic spherical equivalent refraction of $0.80 \pm 1.1 \mathrm{D}$ in boys and $0.81 \pm 1.4$ in girls $(\mathrm{p}=0.85$; Table 1$)$. Girls had a shorter mean axial length than boys $(22.8 \pm 0.8 \mathrm{~mm}$ versus $23.4 \pm 0.8$; $\mathrm{p}<0.001)$, shallower anterior chambers $(3.57 \pm 0.2 \mathrm{~mm}$ versus $3.65 \pm 0.3$; $\mathrm{p}<0.001)$ and steeper corneal radii of curvature $\quad(7.71 \pm 0.2 \quad$ versus $7.85 \pm 0.3 ; \mathrm{p}<0.001)$. The subfoveal choroid was $17 \mu \mathrm{m}$ thicker in girls $(298 \pm 60.6 \mu \mathrm{m} ;$ range $136-485 \mu \mathrm{m})$ than in boys $(281 \pm 55.0 \mu \mathrm{m}$; range 130-463 $\mu \mathrm{m} ; \mathrm{p}<0.001$; Table 1), with a difference of $9.1 \mu \mathrm{m}$ persisting after adjustment for age, ethnicity and axial length $(p=0.017$; Table 2).

Subfoveal choroidal thickness decreased with increasing ocular axial length $(-16.2 \mu \mathrm{m} / \mathrm{mm}, 95 \%$ CI -21.2 to $-12.4, \mathrm{p}<0.001$; Table 2; Fig. 2) and with increasing myopic refraction $(10.0 \mu \mathrm{m} / \mathrm{D} ; \quad 95 \% \quad$ CI $\quad 6.8-13.1$; $\mathrm{p}<0.001$, adjusted for age, ethnicity, axial length and sex; Table 2) while it increased with increasing body height $(1.3 \mu \mathrm{m} / \mathrm{cm} \quad 95 \% \quad$ CI $\quad 0.8-1.9$,

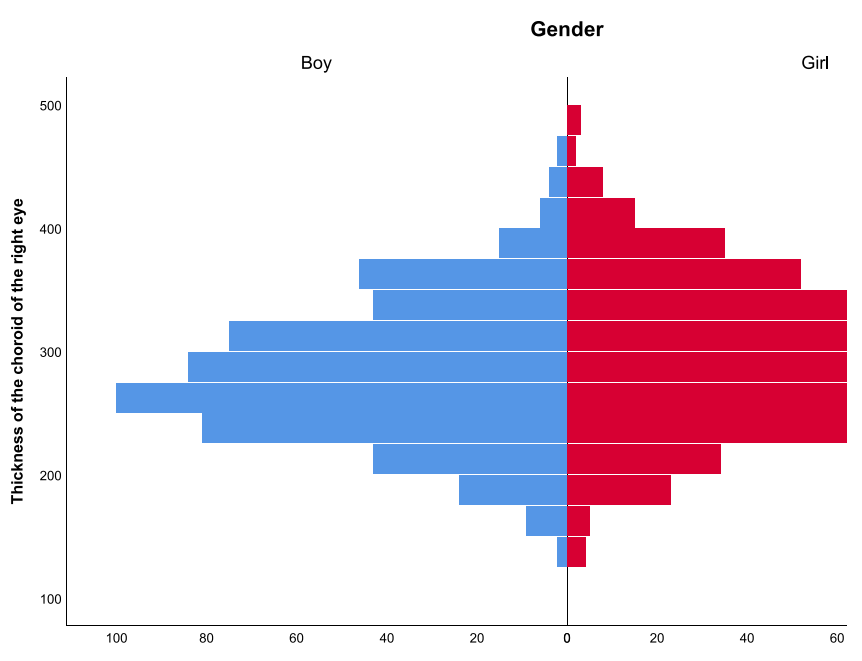

Fig. 2. The distribution of the choroidal thickness in girls (blue) and boys (green).

Table 1. Demographic and clinical characteristics of 1018 study participants from Generation R

\begin{tabular}{lccc}
\hline Parameter & Boys, $n=484(47.5 \%)$ & Girls, $n=534(52.5 \%)$ & $\mathrm{p}^{*}$ \\
\hline European/non-European, $n,(\%)$ & $354 / 130(73 / 27)$ & $384 / 150(72 / 28)$ & \\
Age, y & $9.9(0.3)$ & $9.8(0.3)$ & 0.02 \\
Refractive error, D & $0.80(1.1)$ & $0.81(1.4)$ & 0.85 \\
Axial length, mm & $23.4(0.8)$ & $22.8(0.8)$ & $<0.001$ \\
Anterior chamber, mm & $3.65(0.3)$ & $3.57(0.2)$ & $<0.001$ \\
Corneal radius of curvature, mm & $7.85(0.3)$ & $7.71(0.2)$ & $<0.001$ \\
Height, cm & $142.6(6.5)$ & $141.8(6.5)$ & 0.05 \\
Weight, kg & $35.7(6.6)$ & $36.1(7.9)$ & 0.44 \\
Body mass index, kg/m ${ }^{2}$ & $17.50(2.5)$ & $17.84(3.0)$ & 0.05 \\
Subfoveal choroidal thickness, $\mu \mathrm{m}$ & $281(55.0)$ & $298(60.6)$ & $<0.001$ \\
Birthweight, g & $3535(520)$ & $4392(528)$ & $<0.001$ \\
Gestational age, weeks & $40(1.6)$ & $-0.02(1.0)$ & 0.35 \\
Size for gestational age & $-0.01(1.0)$ & & 0.93 \\
\hline
\end{tabular}

Values are mean and standard deviation (SD).

$\mathrm{D}=$ dioptres, $\mathrm{y}=$ years.

* Paired $t$-test.

${ }^{\dagger}$ Index of birthweight to birth length normalized for sex and gestational age in newborn infants.

$\mathrm{p}<0.001)$. Additionally, choroidal thickness increased with increasing birthweight $\quad(13.0 \mu \mathrm{m} / \mathrm{kg} ; 95 \%$ CI $0.006-0.020 ; \mathrm{p}<0.001)$ and increasing size for gestational age $(8.2 \mu \mathrm{m} / \mathrm{kg}$; 95\% CI 4.6-11.8; $\mathrm{p}<0.001$; confirmed in analysis including gestational age, size for gestational age and birthweight at the same time, Table 3).

Information about maternal smoking habits during pregnancy collected using a questionnaire was available for 885 children and alcohol consumption data during pregnancy for 784 children (Table 4). Children of mothers who smoked up until the time that the pregnancy became known had a thinner choroid than children from mothers who did not smoke during pregnancy ( $p=0.008)$; the association persisted after adjusting for age, sex, axial length, ethnicity and height $(\mathrm{p}=0.016$; Table 4). There was no detectable association between subfoveal choroidal thickness and alcohol consumption during pregnancy (Table 4).

\section{Discussion}

In this population-based child cohort of 9-year-old children, the subfoveal choroid was $17 \mu \mathrm{m}$ thicker in girls than in boys. Additionally, choroidal thickness increased with increasing birthweight and size for gestational age and higher body height was associated with a thicker subfoveal choroid in both girls and boys.

The strong associations between axial length, refractive error and choroidal thickness found in previous studies of adults and older children were also 
Table 2. Associations with subfoveal choroidal thickness in Generation R children

\begin{tabular}{|c|c|c|c|c|c|c|}
\hline \multirow[b]{2}{*}{ Parameter } & \multicolumn{3}{|l|}{ Crude data } & \multicolumn{3}{|c|}{ Adjusted age, ethnicity axial length and sex } \\
\hline & Coefficient $\left(\mathrm{CI}_{95}\right)$ & $\mathrm{p}$ & $R^{2}$ & Coefficient $\left(\mathrm{CI}_{95}\right)$ & $\mathrm{p}$ & $R^{2}$ \\
\hline Age, $\mu \mathrm{m} / \mathrm{y}$ & $11.1(-2.1$ to 24.3$)$ & 0.10 & 0.002 & $13.0(-1.0$ to 23.6$)$ & 0.05 & 0.070 \\
\hline European/non-European & $-7.1(-15.3$ to 1.0$)$ & 0.09 & 0.003 & $-3.8(-10.6$ to 4.9$)$ & 0.35 & 0.070 \\
\hline Sex, girls versus boys, $\mu \mathrm{m}$ & $16.8(9.6$ to 24.0$)$ & $<0.001$ & 0.020 & $9.1(1.5$ to 16.1$)$ & 0.017 & 0.070 \\
\hline Axial length, $\mu \mathrm{m} / \mathrm{mm}$ & $-18.1(-22.3$ to -13.8$)$ & $<0.001$ & 0.064 & $-16.2(-21.2$ to -12.4$)$ & $<0.001$ & 0.070 \\
\hline Anterior chamber, $\mu \mathrm{m} / \mathrm{mm}$ & $-8.3(-23.1$ to 6.5$)$ & 0.27 & 0.001 & $17.7(1.9$ to 33.6$)$ & 0.03 & 0.084 \\
\hline Corneal Radius of curvature, $\mu \mathrm{m} / \mathrm{mm}$ & $-22.2(-36.0$ to -8.4$)$ & $<0.001$ & 0.010 & $19.5(2.7$ to 36.2$)$ & 0.02 & 0.078 \\
\hline Refractive error, $\mu \mathrm{m} / \mathrm{D}$ & $10.0(6.8$ to 13.2$)$ & $<0.001$ & 0.045 & $10.0(6.8$ to 13.1$)$ & $<0.001$ & 0.070 \\
\hline Body mass index, $\mu \mathrm{m}$ & $-0.5(-1.8$ to 0.8$)$ & 0.44 & 0.001 & $-0.3(-1.6$ to 1.0$)$ & 0.66 & 0.07 \\
\hline Weight, $\mu \mathrm{m} / \mathrm{kg}$ & $0.18(-0.3$ to 0.7$)$ & 0.49 & $<0.001$ & $0.4(-0.1$ to 0.9$)$ & 0.10 & 0.076 \\
\hline Height, $\mu \mathrm{m} / 10 \mathrm{~cm}$ & $0.8(0.3$ to 1.4$)$ & 0.003 & 0.009 & $1.3(0.8$ to 1.9$)$ & $<0.001$ & 0.092 \\
\hline Birthweight, $\mu \mathrm{m} / \mathrm{kg}$ & $6.0(0$ to 13.0$)$ & 0.07 & 0.003 & $13.0(6.0$ to 20.0$)$ & $<0.001$ & 0.086 \\
\hline Gestational age, $\mu \mathrm{m} /$ weeks & $0.7(-1.6$ to 3.0$)$ & 0.54 & $<0.001$ & $1.1(-1.2$ to 3.3$)$ & 0.35 & 0.074 \\
\hline Size for gestational age ${ }^{\dagger}$ & $5.7(2.1$ to 9.4$)$ & 0.002 & 0.009 & $8.2(4.6$ to 11.8$)$ & $<0.0001$ & 0.091 \\
\hline
\end{tabular}

Univariate regression analysis (left) of choroidal thickness in relation to single descriptive variables and multivariate regression analysis (right) of same descriptive parameter, but adjusted for age, ethnicity axial length and sex.

$\dagger$ Index of birthweight to birth length normalized for sex and gestational age in newborn infants.

Table 3. Subfoveal choroidal thickness in relation to birth parameters in Generation R children

\begin{tabular}{lllllll}
\hline & \multicolumn{1}{l}{ Age and sex adjusted } & & & \multicolumn{2}{l}{ Multivariate adjustment } \\
\cline { 2 - 3 } Crude data & Estimate $\left(\mathrm{CI}_{95}\right)$ & $\mathrm{p}$ & & Estimate $\left(\mathrm{CI}_{95}\right)$ & $\mathrm{p}$ \\
\hline Birthweight, kg & $0.009(0.002$ to 0.016$)$ & 0.010 & & $0.01(0.003$ to 0.017$)$ & 0.007 \\
Gestational age, weeks & $1.0(-1.3$ to 3.2$)$ & & 0.395 & & $0.7(-1.5$ to 2.9$)$ & 0.547 \\
Size for gestational age, SDS & $5.8(2.2$ to 9.4$)$ & 0.002 & & $6.5(2.8$ to 10.2$)$ & 0.001 \\
\hline
\end{tabular}

Multivariate adjustment included age, sex, ethnicity, axial length and height.

present in the Generation R cohort (Zhu et al. 2005; Niklasson et al. 1991). Thus, increasing axial length and more myopic refraction was associated with a thinner subfoveal choroid. The association between refraction and choroidal thickness persisted after inclusion of axial length in the analysis, despite the close association between refraction and axial length.

The shift in choroidal thickness of $-16.2 \mu \mathrm{m}$ per $\mathrm{mm}$ increase in axial length in 9-year-old children was considerably smaller than the $-28.1 \mu \mathrm{m}$ per $\mathrm{mm}$ found in 11- to 12-year-old children in the CCC2000 Eye Study ( $\mathrm{Li}$ et al. 2014a,b) and far lower than the $-58.2 \mu \mathrm{m}$ per $\mathrm{mm}$ observed in 20 - to 33 -year-old adults (Li et al. 2011). Nevertheless, this cross-study comparison may be seen as evidence that the steepness of the relation increases as the ocular length distribution expands when children grow older. This phenomenon may be important for myopia risk prediction, and it therefore needs further consideration in prospective studies. It should be noted that there may be a systematic offset between the present study's use of cycloplegia and swept-source optical coherence tomography (SS-OCT) instead of the CCC2000's use of spectral-domain optical coherence tomography (SD-OCT) without cycloplegia. The SS-OCT instrument been reported to find an approximately $20-\mu \mathrm{m}$-thinner choroid than the SD-OCT instrument (Philip et al. 2016). Cycloplegia has been reported to increase choroidal thickness (Öner et al.).

In adults, men have a thicker choroid than women (Zhu et al. 2005; Li et al. 2014a,b; Zeng et al. 2012; Gupta et al. 2015). In the present study of children aged 9 years, girls had thicker choroids than boys. In the CCC2000 Eye Study of children aged 11-12 years, girls who had entered puberty had thicker choroids than boys and less developed girls. This may suggest a potential effect of sex or growth hormones on the development of the choroid (Liu et al. 2000). This is supported by animal studies which have reported that insulin-like growth factor 1 (IGF-1) promotes both

Table 4. Subfoveal choroidal thickness in relation to maternal smoking and alcohol consumption during pregnancy.

\begin{tabular}{|c|c|c|c|c|c|}
\hline & \multirow[b]{2}{*}{ Mean choroidal thickness $(\mu \mathrm{m})$} & \multicolumn{2}{|l|}{ Age and sex adjusted } & \multicolumn{2}{|l|}{ Multivariate adjustment } \\
\hline & & Estimate $\left(\mathrm{CI}_{95}\right)$ & $\mathrm{p}$ & Estimate $\left(\mathrm{CI}_{95}\right)$ & $\mathrm{p}$ \\
\hline \multicolumn{6}{|l|}{ Maternal smoking during pregnancy, $n=885$} \\
\hline Smoked until pregnancy was known, $n=73$ & $274(63)$ & $-19(-33$ to -5$) \mu \mathrm{m}$ & 0.008 & $-17(-30$ to -3$) \mu \mathrm{m}$ & 0.01 \\
\hline Never smoked during pregnancy, $n=680$ & $292(59)$ & Reference & & Reference & \\
\hline $\begin{array}{l}\text { Continued smoking in pregnancy, } n=132 \\
\text { Maternal alcohol during pregnancy, } n=784\end{array}$ & $284(53)$ & $-9(-19$ to 2$) \mu \mathrm{m}$ & 0.120 & $-7(-18$ to 3$) \mu \mathrm{m}$ & 0.180 \\
\hline Alcohol until pregnancy was known, $n=98$ & $286(61)$ & $-1(-13$ to 13$) \mu \mathrm{m}$ & 0.994 & $-2(-15$ to 11$) \mu \mathrm{m}$ & 0.713 \\
\hline Never alcohol in pregnancy, $n=307$ & $287(56)$ & Reference & & Reference & \\
\hline Alcohol continued in pregnancy, $n=379$ & $291(60)$ & $-7(-3$ to 14$) \mu \mathrm{m}$ & 0.210 & $1(-8$ to 10$) \mu \mathrm{m}$ & 0.806 \\
\hline
\end{tabular}

Multivariate adjustment, adjusted for age, sex, axial length, ethnicity, height and birthweight. 
elongation of the eye and thickening of the choroid (Penha et al. 2011; Zhu \& Wallman 2009).

Previous studies have found a correlation between growth spurt, pubertal development and myopia, indicating that myopic children and young adults were more likely to be taller, heavier and more physically mature compared to age-matched controls (Yip et al. 2012). However, others found little or no effect of height or growth spurt (Northstone et al. 2013).

Subfoveal choroidal thickness increased with increasing birthweight and size for gestational age, in agreement with the findings of the CCC2000 Eye Study, thus supporting the notion that intrauterine growth conditions have lasting effects on choroidal thickness.

A link with myopia was suggested by the British 1958 cohort which reported that adult myopia was associated with low birthweight for gestational age and maternal smoking in early pregnancy (Rahi et al. 2011). According to an analysis of the associations of active and passive smoking in the Generation R study (Jaddoe et al. 2008), there was a significant association of active maternal smoking with low birthweight and preterm birth. Cessation of active smoking after pregnancy had been confirmed was associated with a higher birthweight compared with continued smoking.

An analysis of the associations of smoking and choroidal thickness comparing smoking and nonsmoking mothers found that maternal smoking until pregnancy had been confirmed was associated with a thinner choroid, but there was no association between smoking throughout pregnancy and choroidal thickness. This paradox suggests that the issue should be studied in a metaanalysis with larger power than the present study.

The strengths of the present study include the population-based recruitment and data collection from foetal life onwards. This study is limited by occasional poor definition of the chorioscleral border on the optical coherence tomography images (Gupta et al. 2015) which led to a relatively high number of disqualified images. Nevertheless, the intraclass correlation coefficient for renewed assessment of choroidal thickness was high, indicating uniformity of the scan analysis process.

In conclusion, this study found, in a younger group of children than previously studied, that subfoveal choroidal thickness increased with increasing birthweight and with increasing size for gestational age. We also found a thinner choroid at the age of 9 years than in a comparable study of 11- to 12-year-old children and a narrower distribution of axial lengths. Future follow-up of the cohort may possibly reveal the extent to which these anatomical effects have an impact on the development of refraction.

\section{References}

Fredrick DR (2002): Myopia. BMJ 324: 1195-1199.

Goldenberg D, Moisseiev E, Goldstein M, Loewenstein A \& Barak A (2012): Enhanced depth imaging optical coherence tomography: choroidal thickness and correlations with age, refractive error, and axial length. Ophthalmic Surg Lasers Imaging 43: 296-301.

Gupta P, Jing T, Marziliano P, et al. (2015): Distribution and determinants of choroidal thickness and volume using automated segmentation software in a population-based study. Am J Ophthalmol 159: 293-301.

Jaddoe VW1, Troe EJ, Hofman A, Mackenbach JP, Moll HA, Steegers EA \& Witteman JC. (2008): Active and passive maternal smoking during pregnancy and the risks of low birthweight and preterm birth: the Generation R Study. Paediatr Perinat Epidemiol 22: 162-171.

Kooijman MN, Kruithof CJ, van Duijn CM, et al. (2016): The Generation R Study: design and cohort update 2017. Eur J Epidemiol 31: 1243-1264.

Li XQ, Larsen M \& Munch IC (2011): Subfoveal choroidal thickness in relation to sex and axial length in 93 Danish university students. Invest Ophthalmol Vis Sci 52: 84388441.

Li XQ, Larsen M, Munkholm A \& Munch IC. (2014a): Choroidal thickness in relation to birth parameters in 1112 year-old children: The Copenhagen Child Cohort 2000 Eye Study. Invest Ophthalmol Vis Sci 56: 617-624.

Li XQ, Jeppesen P, Larsen M \& Munch IC. (2014b): Subfoveal choroidal thickness in 1323 children aged 1112 years and association with puberty: The Copenhagen Child Cohort 2000 Eye Study. Invest Ophthalmol Vis Sci 55: 550-555.

Liu YX, Wikland KA \& Karlberg J (2000): New reference for the age at childhood onset of growth and secular trend in the timing of puberty in Swedish. Acta Paediatr 89: 637-643.

Nickla DL \& Wallman J (2010): The multifunctional choroid. Prog Retin Eye Res 29: 144-168.

Niklasson A, Ericson A, Fryer J.G, Karlberg J, Lawrence C \& Karlberg P (1991): An update of the Swedish reference standards for weight, length and head circumference at birth for given gestational age (1977-1981). Acta Paediatr Scand 80: 756-762.

Northstone K, Guggenheim JA, Howe LD, et al. (2013): Body stature growth trajectories during childhood and the development of myopia. Ophthalmology 120: 1064-1073. e1061.

Penha AM, Schaeffel F \& Feldkaemper M (2011): Insulin, insulin-like growth factor-1, insulin receptor, and insulinlike growth factor-1 receptor expression in the chick eye and their regulation with imposed myopic or hyperopic defocus. Mol Vis 17: 1436-1448.

Philip AM, Gerendas BS, Zhang L et al. (2016): Choroidal thickness maps from spectral domain and swept source optical coherence tomography: algorithmic versus ground truth annotation. Br J Ophthalmol 100: 1372-1376.
Rahi JS, Cumberland PM \& Peckham CS (2011): Myopia over the lifecourse: prevalence and early life influences in the 1958 British birth cohort. Ophthalmology 118: 797-804.

Read SA, Collins MJ, Vincent SJ \& Alonso-Caneiro D (2013a): Choroidal thickness in myopic and nonmyopic children assessed with enhanced depth imaging optical coherence tomography. Invest Ophthalmol Vis Sci 54: 757-886.

Read SA, Collins MJ, Vincent SJ \& Alonso-Caneiro D. (2013b): Choroidal thickness in Childhood. Invest Ophthalmol Vis Sci, 54: 3586-3593.

Resnikoff S, Pascolini D, Mariottia SP \& Pokharel GP (2008): Global magnitude of visual impairment caused by uncorrected refractive errors in 2004. Bull World Health Organ 86: $63-70$.

Yip VC, Pan CW, Lin XY, et al. (2012): he relationship between growth spurts and myopia in Singapore children. Invest Ophthalmol Vis Sci 53: 7961-7966.

Zeng J, Liu R, Zhang XY, et al. (2012): Relationship between gender and posterior pole choroidal thickness in normal eyes [in Chinese]. Zhonghua Yan Ke Za Zhi 48: 1093-1096.

Zhu X \& Wallman J (2009): Opposite effects of glucagon and insulin on compensation for spectacle lenses in chicks. Invest Ophthalmol Vis Sci 50: 24-36.

Zhu X, Park TW, Winawer J \& Wallman J (2005): In a matter of minutes, the eye can know which way to grow. Invest Ophthalmol Vis Sci 46: 2238-2241.

Received on October 13th, 2017.

Accepted on June 7th, 2019

Correspondence:

Caroline C. W. Klaver, MD, $\mathrm{PhD}$

Erasmus Medical Center NA2808

PO Box 5201

3008 AE Rotterdam

the Netherlands

Email: c.c.w.klaver@erasmusmc.nl

The Generation R Study is conducted by the Erasmus Medical Centre in close collaboration with the School of Law and Faculty of Social Sciences of the Erasmus University Rotterdam; the Municipal Health Service Rotterdam area, Rotterdam; the Rotterdam Homecare Foundation, Rotterdam; and the Stichting Trombosedienst \& Artsenlaboratorium Rijnmond (STAR-MDC), Rotterdam. We gratefully acknowledge the contribution of children and parents, general practitioners, hospitals, midwives and pharmacies in Rotterdam. The Generation R Study was made possible by financial support from the Erasmus Medical Centre, Rotterdam; the Erasmus University Rotterdam; the Netherlands Organisation of Scientific Research (NWO); the Netherlands Organization for the Health Research and Development (ZonMw); the Ministry of Education, Culture and Science; the Ministry for Health, Welfare and Sports; the European Commission (DG XII); and the Stichting UitZicht (Grant 2013-24). The study was also supported by the following foundations: MaculaFonds, Novartis Fonds, ODAS, LSBS, Oogfonds and ANVVB that contributed through UitZicht (grant 2014-38). The first author was supported by the Bagenkop-Nielsen Foundation. The sponsors or funding organizations provided unrestricted grants and had no role in the design or conduct of this research. Funding for work on this project at the Rigshospitalet has been contributed by Horizon 2020, the European Union's Framework Programme for Research and Innovation, under project ID 780989 (MERLIN). 